\title{
Analytic three-loop static potential
}

\author{
Roman N. Lee, ${ }^{1}$ Alexander V. Smirnov, ${ }^{2}$ Vladimir A. Smirnov, ${ }^{3}$ and Matthias Steinhauser ${ }^{4}$ \\ ${ }^{1}$ Budker Institute of Nuclear Physics, 630090 Novosibirsk, Russia \\ ${ }^{2}$ Research Computing Center, Moscow State University, 119992 Moscow, Russia \\ ${ }^{3}$ Skobeltsyn Institute of Nuclear Physics of Moscow State University, 119992 Moscow, Russia \\ ${ }^{4}$ Institut für Theoretische Teilchenphysik, Karlsruhe Institute of Technology (KIT), D-76128 Karlsruhe, Germany
}

\begin{abstract}
We present analytic results for the three-loop static potential of two heavy quarks. The analytic calculation of the missing ingredients is outlined and results for the singlet and octet potential are provided.
\end{abstract}

PACS numbers: 12.38.Bx, 14.65.Dw, 14.65.Fy, 14.65.Ha

\section{INTRODUCTION}

The static potential between two heavy quarks belongs to the fundamental quantities of QCD. In lowest order it is described by the Coulomb potential adapted to QCD. Such an approach has already been used more than 40 years ago 1] to describe the bound state of heavy quarks. Shortly afterwards the one-loop corrections were computed [2, 3] and the two-loop terms were added towards the end of the nineties [4 6$]$. Light quark mass effects at two loops can be found in Ref. [7]. About eight years ago the three-loop corrections have been computed by two groups in Refs. [8 10]. However, in contrast to the lower-order expressions, the three-loop results could only be presented in numerical form. In fact, in Refs. 8, 9] three coefficients in the expansion of the master integrals around $d=4$, where $d$ is the space-time dimension, could only be evaluated numerically (see also below). The evaluation of one of them is described in detail in Ref. 11] (in a broader context) and the remaining two coefficients are considered in Section $\amalg$ of this paper. We are thus in the position to present analytic results at three loops. The corresponding expressions can be found in Section III]

A generalization of the three-loop singlet potential has been considered in Ref. [12]. It is still assumed that the heavy colour sources form a singlet state, however, the colour representation is kept general.

The new results can also be used to present analytic expressions for the so-called octet potential which describes the situation where the quark and anti-quark do not form a colour-singlet but a colour-octet state. Two- and (numerical) three-loop results have been obtained in Refs. [13, 14] and [15], respectively. Analytic results for the octet potential are presented in Section IV.

In order to fix the notation we write the momentum space potential in the form

$$
V^{[c]}(|\vec{q}|)=-4 \pi C^{[c]} \frac{\alpha_{s}(|\vec{q}|)}{\vec{q}^{2}}\left[1+\frac{\alpha_{s}(|\vec{q}|)}{4 \pi} a_{1}^{[c]}+\left(\frac{\alpha_{s}(|\vec{q}|)}{4 \pi}\right)^{2} a_{2}^{[c]}+\left(\frac{\alpha_{s}(|\vec{q}|)}{4 \pi}\right)^{3}\left(a_{3}^{[c]}+8 \pi^{2} C_{A}^{3} \ln \frac{\mu^{2}}{\vec{q}^{2}}\right)+\cdots\right],
$$

with $C^{[1]}=C_{F}$ for the colour-singlet and $C^{[8]}=C_{F}-C_{A} / 2$ for the colour-octet case. Here, $C_{A}=N_{c}$ and $C_{F}=$ $\left(N_{c}^{2}-1\right) /\left(2 N_{c}\right)$ are the eigenvalues of the quadratic Casimir operators of the adjoint and fundamental representations of the $\mathrm{SU}\left(N_{c}\right)$ colour gauge group, respectively. The strong coupling $\alpha_{s}$ is defined in the $\overline{\mathrm{MS}}$ scheme and for the renormalization scale we choose $\mu=|\vec{q}|$ in order to suppress the corresponding logarithms. The general results, both in momentum and coordinate space, can, e.g., be found in Appendix A of Ref. [15].

The logarithmic term in Eq. (10) has its origin in an infra-red divergence which is present for the first time at three loops as has been pointed out in Ref. [16]. The corresponding pole has been subtracted minimally. Its presence can be understood in the context of methods of regions and potential non-relativistic QCD [17 21] where $V^{[c]}$ appears as a matching coefficient. Thus, the infrared divergence cancels against ultraviolet divergences of the ultrasoft contributions. The latter have been studied in Refs. 20, 22, 23]. For the resummation of leading and next-to-leading ultrasoft logarithms we refer to [24 26].

The three-loop coefficient $a_{3}$ only has a moderate numerical value (see, e.g., discussion in Ref. [9]) and has thus only a relative small influence on phenomenological quantities. This is in contrast to the two-loop coefficient which is of the same order of magnitude as $a_{1}$. However, since the static potential is a matching coefficient, it is hence not a physical quantity. In fact, $a_{3}$ is scheme dependent and only the combination with all other building blocks leads to meaningful quantities.

For later convenience we decompose the three-loop corrections according to the number of closed fermion loops

$$
a_{3}^{[c]}=a_{3}^{[c],(3)} n_{l}^{3}+a_{3}^{[c],(2)} n_{l}^{2}+a_{3}^{[c],(1)} n_{l}+a_{3}^{[c],(0)},
$$




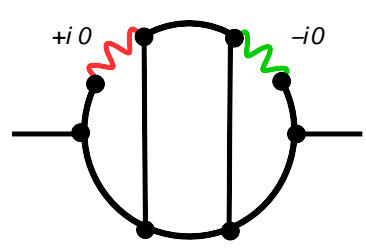

(a) $I_{11}$

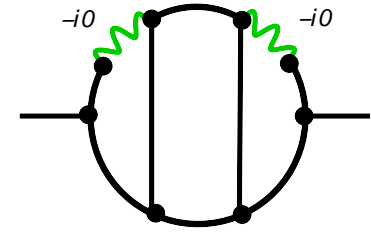

(b) $I_{16}$

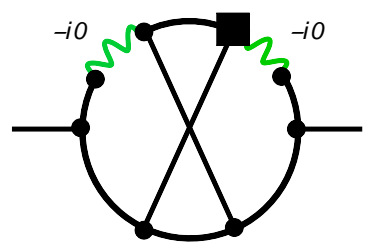

(c) $I_{18}$

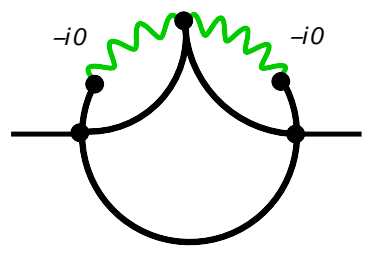

(d) $I_{14}$

FIG. 1: (a)-(c): Master integrals entering $a_{3}^{[c]}$ which were only known numerically. Solid lines denote relativistic scalar propagators and wavy lines refer to static propagators. For the latter the causality prescription is given explicitly where $\pm i 0$ indicates a propagator of the form $1 /\left(-k_{0} \pm i 0\right)$ with $k_{0}$ being the zeroth component of the momentum flowing through the corresponding line. The square in $I_{18}$ indicates a convenient choice for the numerator which is specified in Ref. [11]. $I_{18}$ is finite and only the $\mathcal{O}\left(\epsilon^{0}\right)$ term is needed. For $I_{11}$ and $I_{16}$ also the $\mathcal{O}\left(\epsilon^{1}\right)$ terms enter $a_{3}^{[c]}$. (d): Master integral which is needed for the computation of the integrals in (b) and (c). The integral $I_{15}$ belongs to the same integral family as $I_{14}$, however, has an additional dot on the lower line.

where $n_{l}$ is the number of light (massless) quarks. We furthermore consider the difference between the singlet and octet contributions and write $(i=0,1,2,3)$

$$
a_{3}^{[8],(i)}=a_{3}^{[1],(i)}+\delta a_{3}^{[8],(i)} .
$$

In Section IV we provide analytical results for $\delta a_{3}^{[8],(i)}$.

The three-loop coefficient of the colour singlet potential, $a_{3}^{[1]}$, has entered a number of physical applications as building block (see also Ref. [27] for a recent review on applications of non-relativistic QCD to high-energy processes). To name a few of them we want to mention the next-to-next-to-next-to-leading order corrections to the leptonic decay width of the $\Upsilon(1 S)$ meson [28] and the top quark threshold production in electron positron colliders [29]. Furthermore, $a_{3}$ has entered analyses to determine precise values for the charm and bottom quark masses [30 33] and the strong coupling constant [34].

\section{CALCULATION OF $I_{11}$ AND $I_{16}$}

The calculation of $a_{3}^{[1]}$ as performed in [9] requires the evaluation of 41 master integrals which can be sub-divided into three different classes: There are ten integrals which do not have any static line (i.e. a propagator of the form $1 /\left(-k_{0} \pm i 0\right)$, see also Fig. 1), and are thus known since long. Furthermore, we have 14 integrals with a massless oneloop insertion. They can easily be integrated in terms of $\Gamma$ functions using standard techniques. The corresponding results have been presented in Ref. [35]. Results for 16 more complicated integrals can be found in Ref. [36] as expansions in $\epsilon=(4-d) / 2$ to the necessary order except for two integrals ( $I_{11}$ and $I_{16}$ of Ref. [36], see also Fig. 11(a) and (b)). Their $\mathcal{O}(\epsilon)$ terms enter $a_{3}^{[1]}$, however, they were only known numerically. The evaluation of these coefficients will be described in the remainder of this section. For completeness we want to mention that the third numerical ingredient required in [9] comes from the finite diagram in Fig. 1(c) (the $41^{\text {th }}$ master integral) which has been computed in a parallel article [11].

Let us also mention that techniques which have been used to compute master integrals in [10] can be found in Ref. 37], see also 38] for a status report of the approach used in Ref. 10].

The method which is used to compute $I_{11}$ and $I_{16}$ is based on the dimensional recurrence relation and analyticity with respect to space-time dimensionality $d$ (the so-called "DRA method") and has been developed in Ref. [39]. In Ref. [40] this method has been applied for the first time to the case with more than one master integral in a sector. Some integrals taken from families of integrals for the three-loop static quark potential and denoted in [40] by $I_{14}$ and $I_{15}$ (see Fig. $1(\mathrm{~d})$ ) have been calculated. Note that $I_{14}$ and $I_{15}$ are the only nontrivial integrals entering the right-hand side of the dimensional recurrence relation for $I_{16}$. Therefore, in principle, the results of Ref. [40] make the calculation of $I_{16}$ straightforward.

However, the numerical issues related to the calculation of contributions to the inhomogeneous terms proportional to $I_{14}$ and $I_{15}$ in the right-hand side of dimensional recurrence relations for $I_{16}$ are quite involved. The most complicated 
part of this contribution has the form

$$
T(\nu)=\sum_{k=0}^{\infty} v^{T}(\nu+k) \sum_{n=k}^{\infty}\left(\prod_{l=k}^{n} \mathrm{M}(\nu+l)\right) u(\nu+n),
$$

where $\nu=d / 2, v^{T}(x), \mathrm{M}(x)$, and $u(x)$ are a row-vector, a $2 \times 2$ matrix and a column-vector, respectively. Their components are rational functions of the variable $x$. In order to calculate the sums in Eq. (4) without nested loops, we apply the standard trick of the DRA method, see Ref. [41]. Namely, let us denote

$$
F(k)=\sum_{n=k}^{\infty} \mathrm{P}(k, n) u(\nu+n),
$$

where $\mathrm{P}(k, n)=\prod_{l=k}^{n} \mathrm{M}(\nu+l)$. Then

$$
T(\nu)=\sum_{k=0}^{\infty} v^{T}(\nu+k) F(k) .
$$

Using Eq. (5), the function $F(k)$ can be calculated for given $k$ in one loop if one takes into account the recurrence relation $\mathrm{P}(k, n+1)=\mathrm{P}(k, n) \mathrm{M}(\nu+n+1)$. Now we note that $F(k)$ satisfies the recurrence relation

$$
F(k+1)=\mathrm{M}^{-1}(\nu+k) F(k)-u(\nu+k) .
$$

Therefore, in order to calculate consecutive terms of the sum in Eq. (6) we need to use Eq. (5) only once, and then use the recurrence relation (7). However, the price we have to pay is much higher than for scalar sums. This is connected with the multiplication by the inverse matrix $\mathrm{M}^{-1}(\nu+k)$. For $x \rightarrow \infty$ the elements of $\mathrm{M}(x)$ are of order unity, while its determinant tends to $1 / 1024$. Due to this fact, the multiplication by $\mathrm{M}^{-1}$ involves large cancellations which result in rapid precision loss. For example, using a precision of 7000 digits in the initial expression we obtain only about 370 digits in the final result.

Besides, it appears that the sum over $n$ in the definition of $F(k)$ converges very slowly, with the summand behaving as $n^{-\alpha}(\alpha>1)$ at large $n$. So, in order to obtain the high-precision numerical result suitable for using PSLQ [42], one has to apply the matrix analog of the convergence acceleration algorithm described in Ref. [43]. In particular, one needs to know the exponent $\alpha$ of the power-like decay. This appears to be possible thanks to Ref. [44], where a method for finding the asymptotic behaviour of the solutions of recurrence relations was developed. Once we dealt with these numerical issues, we have obtained the result ${ }^{1}$

$$
\begin{aligned}
I_{16}= & -\frac{56 \pi^{4}}{135 \epsilon}-\left(\frac{112 \pi^{4}}{135}+\frac{16 \pi^{2} \zeta(3)}{9}+\frac{8 \zeta(5)}{3}\right)+\left(\frac{968 \zeta(5)}{3}-16 \pi^{4} l_{2}+\frac{136 \zeta(3)^{2}}{3}+\frac{400 \pi^{2} \zeta(3)}{9}-\frac{838 \pi^{6}}{2835}\right. \\
& \left.+\frac{1792 \pi^{4}}{135}\right) \epsilon+\left(\frac{6144 s_{6} l_{2}}{7}-\frac{6144 s_{7} \mathrm{a}}{7}+\frac{15360 s_{7 \mathrm{~b}}}{7}+1536 \alpha_{4} \zeta(3)+1024 \pi^{2} \alpha_{5}-256 \pi^{2} \alpha_{4}-\frac{64}{9} \pi^{4} l_{2}^{3}\right. \\
& -2976 \zeta(5) l_{2}^{2}-64 \pi^{2} \zeta(3) l_{2}^{2}-\frac{112}{3} \pi^{4} l_{2}^{2}-\frac{7680 \zeta(3)^{2} l_{2}}{7}-\frac{544 \pi^{6} l_{2}}{315}+128 \pi^{4} l_{2}+\frac{306202 \zeta(7)}{21}-\frac{12182 \pi^{2} \zeta(5)}{7} \\
& \left.+\frac{64 \zeta(5)}{3}-\frac{1168 \zeta(3)^{2}}{3}-\frac{11828 \pi^{4} \zeta(3)}{945}+\frac{1664 \pi^{2} \zeta(3)}{9}+\frac{1376 \pi^{6}}{135}-\frac{12544 \pi^{4}}{135}+768 s_{6}\right) \epsilon^{2}+O\left(\epsilon^{3}\right),
\end{aligned}
$$

where $\zeta(n)$ is Riemann's zeta function evaluated at $n$ and

$$
\begin{aligned}
l_{2} & =\log (2), \\
\alpha_{n} & =\operatorname{Li}_{n}(1 / 2)+\frac{(-\log 2)^{n}}{n !}, \\
s_{6} & =\zeta(-5,-1)+\zeta(6), \\
s_{7 a} & =\zeta(-5,1,1)+\zeta(-6,1)+\zeta(-5,2)+\zeta(-7), \\
s_{7 b} & =\zeta(7)+\zeta(5,2)+\zeta(-6,-1)+\zeta(5,-1,-1) .
\end{aligned}
$$

\footnotetext{
${ }^{1}$ See Fig. 1 b) for a graphical definition and Eq. (4.1) of Ref. [40] normalization factors.
} 
$\zeta\left(m_{1}, \ldots, m_{k}\right)$ are multiple zeta values given by

$$
\zeta\left(m_{1}, \ldots, m_{k}\right)=\sum_{i_{1}=1}^{\infty} \sum_{i_{2}=1}^{i_{1}-1} \cdots \sum_{i_{k}=1}^{i_{k-1}-1} \prod_{j=1}^{k} \frac{\operatorname{sgn}\left(m_{j}\right)^{i_{j}}}{i_{j}^{\left|m_{j}\right|}} .
$$

In order to apply the DRA method to $I_{11}$, one has to take into account that the dimensional recurrence relation for $I_{11}$ contains now two non-trivial integrals denoted in [40] by $I_{9}$ and $I_{10}$. So, in a first step one has to apply the DRA method to these two integrals. Fortunately, they can be calculated along the same lines as $I_{14}$ and $I_{15}$ from which they differ only by the $\pm i 0$ prescription in one of the linear denominators. In particular, the summing factor has the same form as in Ref. [40] (see Eq. (4.14) of that paper). Plugging the results for $I_{9}$ and $I_{10}$ in the dimensional recurrence relation for $I_{11}$ and applying the DRA method, we obtain ${ }^{2}$

$$
\begin{aligned}
I_{11}= & \frac{64 \pi^{4}}{135 \epsilon}+\left(\frac{128 \pi^{4}}{135}+\frac{32 \pi^{2} \zeta(3)}{9}-\frac{8 \zeta(5)}{3}\right)+\left(16 \pi^{4} l_{2}+\frac{968 \zeta(5)}{3}+\frac{136 \zeta(3)^{2}}{3}-\frac{800 \pi^{2} \zeta(3)}{9}+\frac{548 \pi^{6}}{2835}-\frac{2048 \pi^{4}}{135}\right) \epsilon \\
& +\left(\frac{6144 s_{6} l_{2}}{7}-\frac{6144 s_{7 \mathrm{a}}}{7}+\frac{15360 s_{7 \mathrm{~b}}}{7}+1536 \alpha_{4} \zeta(3)-2048 \pi^{2} \alpha_{5}+512 \pi^{2} \alpha_{4}-\frac{64}{9} \pi^{4} l_{2}^{3}-2976 \zeta(5) l_{2}^{2}\right. \\
& -64 \pi^{2} \zeta(3) l_{2}^{2}+\frac{80}{3} \pi^{4} l_{2}^{2}-\frac{7680 \zeta(3)^{2} l_{2}}{7}-\frac{208 \pi^{6} l_{2}}{315}-128 \pi^{4} l_{2}+\frac{306202 \zeta(7)}{21}+\frac{1482 \pi^{2} \zeta(5)}{7}+\frac{64 \zeta(5)}{3} \\
& \left.-\frac{1168 \zeta(3)^{2}}{3}-\frac{70208 \pi^{4} \zeta(3)}{945}-\frac{3328 \pi^{2} \zeta(3)}{9}-\frac{1504 \pi^{6}}{135}+\frac{14336 \pi^{4}}{135}+768 s_{6}\right) \epsilon^{2}+O\left(\epsilon^{3}\right) .
\end{aligned}
$$

Note that the $\mathcal{O}\left(\epsilon^{2}\right)$ terms of $I_{16}$ and $I_{11}$ in Eqs. (8) and (11) are not needed for $a_{3}^{[c]}$. We nevertheless provide these results to demonstrate the powerfulness of the DRA method.

In principle, the DRA method is also applicable to the calculation of $I_{18}$. However, the difficulties related to the slow convergence of certain matrix sums and the corresponding precision loss appear to be overwhelming. For this reason, the method of differential equations has been applied to $I_{18}$, see Ref. [11].

\section{SINGLET POTENTIAL}

In this Section we present analytic expressions for $a_{3}^{[1]}$. One- and two-loop results using the same notation can be found in Ref. [15]. Analytic results for the coefficients of $n_{l}^{3}$ and $n_{l}^{2}$ have already been presented in Ref. [8]. Here, they are repeated for completeness

$$
\begin{aligned}
& a_{3}^{[1],(3)}=-\left(\frac{20}{9}\right)^{3} T_{F}^{3}, \\
& a_{3}^{[1],(2)}=\left(\frac{12541}{243}+\frac{368 \zeta(3)}{3}+\frac{64 \pi^{4}}{135}\right) C_{A} T_{F}^{2}+\left(\frac{14002}{81}-\frac{416 \zeta(3)}{3}\right) C_{F} T_{F}^{2} .
\end{aligned}
$$

Let us now turn to the $n_{l}^{1}$ and $n_{l}^{0}$ term. Expressed in terms of the eigenvalues of the Casimir operators and higher order group invariants $d_{F}^{a b c d}$ and $d_{A}^{a b c d}$ (see, e.g., Ref. [45]) we obtain for the linear- $n_{l}$ term the analytic result

$$
\begin{aligned}
a_{3}^{[1],(1)}= & \frac{d_{F}^{a b c d} d_{F}^{a b c d}}{N_{A}}\left\{\pi^{2}\left(\frac{1264}{9}-\frac{976 \zeta(3)}{3}+l_{2}(64+672 \zeta(3))\right)+\pi^{4}\left(-\frac{184}{3}+\frac{32 l_{2}}{3}-32 l_{2}^{2}\right)+\frac{10 \pi^{6}}{3}\right\} \\
& +T_{F}\left\{C_{F}^{2}\left(\frac{286}{9}+\frac{296 \zeta(3)}{3}-160 \zeta(5)\right)+C_{A} C_{F}\left(-\frac{71281}{162}+264 \zeta(3)+80 \zeta(5)\right)\right. \\
& +C_{A}^{2}\left[-\frac{58747}{486}+\pi^{2}\left(\frac{17}{27}-32 \alpha_{4}+l_{2}\left(-\frac{4}{3}-14 \zeta(3)\right)-\frac{19 \zeta(3)}{3}\right)-356 \zeta(3)\right. \\
& \left.\left.+\pi^{4}\left(-\frac{157}{54}-\frac{5 l_{2}}{9}+l_{2}^{2}\right)+\frac{1091 \zeta(5)}{6}+\frac{57(\zeta(3))^{2}}{2}+\frac{761 \pi^{6}}{2520}-48 s_{6}\right]\right\}
\end{aligned}
$$

\footnotetext{
${ }^{2}$ See Fig. 11a) for a graphical definition and Eq. (4.1) of Ref. [40] normalization factors.
} 
and the gluonic part is given by

$$
\begin{aligned}
a_{3}^{[1],(0)}= & \frac{d_{F}^{a b c d} d_{A}^{a b c d}}{N_{A}}\left\{\pi^{2}\left[\frac{7432}{9}-4736 \alpha_{4}+l_{2}\left(\frac{14752}{3}-3472 \zeta(3)\right)-\frac{6616 \zeta(3)}{3}\right]\right. \\
& \left.+\pi^{4}\left(-156+\frac{560 l_{2}}{3}+\frac{496 l_{2}^{2}}{3}\right)+\frac{1511 \pi^{6}}{45}\right\}+C_{A}^{3}\left\{\frac{385645}{2916}+\pi^{2}\left[-\frac{953}{54}+\frac{584 \alpha_{4}}{3}+\frac{175 \zeta(3)}{2}\right.\right. \\
& \left.+l_{2}\left(-\frac{922}{9}+\frac{217 \zeta(3)}{3}\right)\right]+\frac{584 \zeta(3)}{3}+\pi^{4}\left(\frac{1349}{270}-\frac{20 l_{2}}{9}-\frac{40 l_{2}^{2}}{9}\right)-\frac{1927 \zeta(5)}{6}-\frac{143(\zeta(3))^{2}}{2} \\
& \left.-\frac{4621 \pi^{6}}{3024}+144 s_{6}\right\} .
\end{aligned}
$$

The numerical evaluation of the analytic results is in full agreement (including all digits) with [8 10].

It is interesting to note that the contributions proportional to $d_{F}^{a b c d} d_{F}^{a b c d}$ and $d_{F}^{a b c d} d_{A}^{a b c d}$ only involve $\pi^{2}, \pi^{4}$ and $\pi^{6}$ terms. Note that these colour structures appear for the first time at three-loop order. On the other hand, the other colour structures basically involve all constants one expects up to transcendentality weight six. Note, however, that the constant $s_{6}$ is only present in the most non-abelian parts, i.e., $T_{F} C_{A}^{2}$ and $C_{A}^{3}$. Let us also mention that $\log (2)$ terms are present to first, second and fourth power but there are no cubic terms.

In a next step we specify to $\mathrm{SU}\left(N_{c}\right)$ and replace the colour factors by

$$
\begin{aligned}
& C_{A}=N_{c}, \quad C_{F}=\frac{N_{c}^{2}-1}{2 N_{c}}, \quad T_{F}=\frac{1}{2}, \quad N_{A}=N_{c}^{2}-1, \\
& \frac{d_{F}^{a b c d} d_{F}^{a b c d}}{N_{A}}=\frac{18-6 N_{c}^{2}+N_{c}^{4}}{96 N_{c}^{2}}, \quad \frac{d_{F}^{a b c d} d_{A}^{a b c d}}{N_{A}}=\frac{N_{c}\left(N_{c}^{2}+6\right)}{48} .
\end{aligned}
$$

This leads to

$$
\begin{aligned}
a_{3}^{[1],(1)}= & \frac{66133}{648}+\pi^{2}\left(-\frac{79}{9}+l_{2}(-4-42 \zeta(3))+\frac{61 \zeta(3)}{3}\right)-\frac{272 \zeta(3)}{3}+\pi^{4}\left(\frac{23}{6}-\frac{2 l_{2}}{3}+2 l_{2}^{2}\right)+20 \zeta(5)-\frac{5 \pi^{6}}{24} \\
& +\frac{1}{N_{c}^{2}}\left\{\frac{143}{36}+\pi^{2}\left[\frac{79}{3}-61 \zeta(3)+l_{2}(12+126 \zeta(3))\right]+\frac{37 \zeta(3)}{3}+\pi^{4}\left(-\frac{23}{2}+2 l_{2}-6 l_{2}^{2}\right)-20 \zeta(5)\right. \\
& \left.+\frac{5 \pi^{6}}{8}\right\}+N_{c}^{2}\left\{-\frac{323615}{1944}+\pi^{2}\left(\frac{16}{9}-16 \alpha_{4}-\frac{59 \zeta(3)}{9}\right)-\frac{299 \zeta(3)}{3}+\pi^{4}\left(-\frac{113}{54}-\frac{l_{2}}{6}+\frac{l_{2}^{2}}{6}\right)\right. \\
& \left.+\frac{1091 \zeta(5)}{12}+\frac{57(\zeta(3))^{2}}{4}+\frac{13 \pi^{6}}{70}-24 s_{6}\right\}, \\
a_{3}^{[1],(0)}= & N_{c}\left\{\pi^{2}\left[\frac{929}{9}-592 \alpha_{4}+l_{2}\left(\frac{1844}{3}-434 \zeta(3)\right)-\frac{827 \zeta(3)}{3}\right]+\pi^{4}\left(-\frac{39}{2}+\frac{70 l_{2}}{3}+\frac{62 l_{2}^{2}}{3}\right)+\frac{1511 \pi^{6}}{360}\right\} \\
& +N_{c}^{3}\left\{\frac{385645}{2916}+\pi^{2}\left(-\frac{4}{9}+96 \alpha_{4}+\frac{374 \zeta(3)}{9}\right)+\frac{584 \zeta(3)}{3}+\pi^{4}\left(\frac{943}{540}+\frac{5 l_{2}}{3}-l_{2}^{2}\right)\right. \\
& \left.-\frac{1927 \zeta(5)}{6}-\frac{143(\zeta(3))^{2}}{2}-\frac{29 \pi^{6}}{35}+144 s_{6}\right\} .
\end{aligned}
$$

Finally, for $N_{c}=3$ we have

$$
\begin{aligned}
a_{3}^{[1],(1)}= & -\frac{452213}{324}+\pi^{2}\left[\frac{274}{27}-\frac{409 \zeta(3)}{9}-144 \alpha_{4}+l_{2}\left(-\frac{8}{3}-28 \zeta(3)\right)\right]-\frac{26630 \zeta(3)}{27} \\
& +\pi^{4}\left(-\frac{293}{18}-\frac{35 l_{2}}{18}+\frac{17 l_{2}^{2}}{6}\right)+\frac{30097 \zeta(5)}{36}+\frac{1931 \pi^{6}}{1260}+\frac{513(\zeta(3))^{2}}{4}-216 s_{6}, \\
a_{3}^{[1],(0)}= & \frac{385645}{108}+\pi^{2}\left[\frac{893}{3}+816 \alpha_{4}+l_{2}(1844-1302 \zeta(3))+295 \zeta(3)\right]+5256 \zeta(3)
\end{aligned}
$$




$$
+\pi^{4}\left(-\frac{227}{20}+115 l_{2}+35 l_{2}^{2}\right)-\frac{17343 \zeta(5)}{2}-\frac{1643 \pi^{6}}{168}-\frac{3861(\zeta(3))^{2}}{2}+3888 s_{6},
$$

which in numerical form is given by

$$
a_{3}^{[1]}=13432.5648565-3289.9052968 n_{l}+185.9900266 n_{l}^{2}-1.3717421 n_{l}^{3} .
$$

\section{OCTET POTENTIAL}

In this Section we proceed similar to the previous one and present results for $\delta a_{3}^{[8],(i)}$ defined in Eq. (3). We discuss the results in terms of $C_{A}, C_{F}$, etc. in Appendix $\mathrm{A}$ and present in this section expressions in terms of $N_{c}$. We have $\delta a_{3}^{[8],(i)}=0$ for $i=2$ and $i=3$ and for the linear- $n_{l}$ and $n_{l}$-independent terms we get

$$
\begin{aligned}
\delta a_{3}^{[8],(1)}= & \pi^{2}\left[-\frac{11}{3}-31 \zeta(3)+l_{2}(4+42 \zeta(3))\right]+\pi^{4}\left(-\frac{7}{6}+\frac{2 l_{2}}{3}-2 l_{2}^{2}\right)+\frac{5 \pi^{6}}{24} \\
& +N_{c}^{2}\left[\pi^{2}\left(\frac{8}{9}+48 \alpha_{4}+25 \zeta(3)\right)+\pi^{4}\left(\frac{2}{3}+\frac{2 l_{2}}{3}\right)-\frac{13 \pi^{6}}{20}\right] \\
\delta a_{3}^{[8],(0)}= & N_{c}^{3}\left\{\pi^{2}\left[\frac{139}{9}+304 \alpha_{4}+15 \zeta(3)+l_{2}\left(-\frac{1844}{3}+434 \zeta(3)\right)\right]\right. \\
& \left.+\pi^{4}\left(\frac{295}{6}-30 l_{2}-\frac{62 l_{2}^{2}}{3}\right)-\frac{1187 \pi^{6}}{360}\right\}
\end{aligned}
$$

which for $N_{c}=3$ leads to

$$
\begin{aligned}
\delta a_{3}^{[8],(1)}= & -\frac{677 \pi^{6}}{120}+\pi^{4}\left(\frac{29}{6}+\frac{20 l_{2}}{3}-2 l_{2}^{2}\right)+\pi^{2}\left[\frac{13}{3}+432 \alpha_{4}+194 \zeta(3)+l_{2}(4+42 \zeta(3))\right], \\
\delta a_{3}^{[8],(0)}= & \pi^{2}\left[417+8208 \alpha_{4}+405 \zeta(3)+l_{2}(-16596+11718 \zeta(3))\right]+\pi^{4}\left(\frac{2655}{2}-810 l_{2}-558 l_{2}^{2}\right) \\
& -\frac{3561 \pi^{6}}{40} .
\end{aligned}
$$

It is interesting to note that $\delta a_{3}^{[8],(0)}$ and $\delta a_{3}^{[8],(1)}$ have an overall factor $\pi^{2}$ which was predicted in Ref. [15] on the basis of the involved master integrals. Although they could not be computed analytically it was possible to show that there is an overall factor $\pi^{2}$, a feature which is also observed at two-loop order in QCD [13, 14] and in $\mathcal{N}=4$ supersymmetric Yang Mills theories [46].

In numerical form we obtain for the complete three-loop coefficient

$$
\delta a_{3}^{[8]}=-2634.7351731+367.9626044 n_{l} .
$$

\section{CONCLUSIONS}

The interaction of a slowly moving heavy quark-anti-quark pair can be described with the help of a static potential, a concept which is familiar from ordinary quantum mechanics. Its perturbative part is obtained from the exchange of soft gluons which are conveniently considered in the framework of non-relativistic QCD. Numerical results for the three-loop potential, which have entered a number of physical observables, have been obtained eight years ago by two independent groups [8 10]. The obtained precision has been sufficient for all physical applications where $a_{3}$ entered as a building block. However, from the aesthetic point of view it is important to obtain analytic results for higher order quantum corrections. This has been achieved in this paper. We have obtained analytic results for the three-loop corrections to the singlet and octet potential which are presented in Sections III and IV respectively.

\section{Acknowledgements}

We thank Alexander Penin for carefully reading the manuscript. R.L. acknowledges support through RFBR grant No. 15-02-07893. 


\section{Appendix A: $\delta a_{2}^{[8]}$ and $\delta a_{3}^{[8]}$ in terms of colour invariants}

In this appendix we present results for $\delta a_{3}^{[8],(1)}$ and $\delta a_{3}^{[8],(0)}$ in terms of $C_{A}, C_{F}, T_{F}, N_{A}, d_{F}^{a b c d}$ and $d_{A}^{a b c d}$. Let us mention that the representation given in Eq. (11) is only valid for $\mathrm{SU}\left(N_{c}\right)$. Thus, in the following we present results for $C^{[8]} \delta a_{3}^{[8],(i)}(i=0,1)$ with $C^{[8]}=C_{F}-C_{A} / 2$. For completeness we also present the two-loop expression; at one-loop order we have $\delta a_{1}^{[8]}=0$. Our results read

$$
\begin{aligned}
& C^{[8]} \delta a_{2}^{[8]}=\left(\frac{\pi^{4}}{12}-\pi^{2}\right)\left(C_{A}^{3}-48 \frac{d_{F}^{a b c d} d_{A}^{a b c d}}{N_{A}}\right) \\
& C^{[8]} \delta a_{3}^{[8],(1)}=C_{A} \frac{d_{F}^{a b c d} d_{F}^{a b c d}}{N_{A}}\left[\pi^{2}\left(\frac{88}{9}-\frac{32 l_{2}}{3}+\frac{248 \zeta(3)}{3}-112 \zeta(3) l_{2}\right)+\pi^{4}\left(\frac{28}{9}-\frac{16 l_{2}}{9}+\frac{16 l_{2}^{2}}{3}\right)-\frac{5 \pi^{6}}{9}\right] \\
& +\frac{d_{F}^{a b c d} d_{A}^{a b c d}}{N_{A}}\left[\pi^{2}\left(\frac{4}{3}-192 \alpha_{4}-\frac{16 l_{2}}{3}-\frac{176 \zeta(3)}{3}-56 l_{2} \zeta(3)\right)+\pi^{4}\left(-\frac{10}{9}-\frac{32 l_{2}}{9}+\frac{8 l_{2}^{2}}{3}\right)+\frac{209 \pi^{6}}{90}\right] \\
& +C_{A}^{3} T_{F}\left[\pi^{2}\left(-\frac{7}{27}+8 \alpha_{4}+\frac{4 l_{2}}{9}+\frac{13 \zeta(3)}{18}+\frac{14 l_{2} \zeta(3)}{3}\right)+\pi^{4}\left(-\frac{1}{54}+\frac{5 l_{2}}{27}-\frac{2 l_{2}^{2}}{9}\right)-\frac{23 \pi^{6}}{270}\right], \\
& C^{[8]} \delta a_{3}^{[8],(0)}=C_{A} \frac{d_{F}^{a b c d} d_{A}^{a b c d}}{N_{A}}\left[\pi^{2}\left(-\frac{2356}{9}+3520 \alpha_{4}-\frac{7376 l_{2}}{3}+1420 \zeta(3)+1736 \zeta(3) l_{2}\right)+\pi^{4}\left(66-\frac{200 l_{2}}{3}-\frac{248 l_{2}^{2}}{3}\right)\right. \\
& \left.-\frac{511 \pi^{6}}{18}\right]+\frac{d_{A}^{a b c d} d_{A}^{a b c d}}{N_{A}}\left[\pi^{2}\left(\frac{50}{3}-\frac{1184 \alpha_{4}}{3}+\frac{3688 l_{2}}{9}-\frac{370 \zeta(3)}{3}-\frac{868 l_{2} \zeta(3)}{3}\right)\right. \\
& \left.+\pi^{4}\left(-\frac{197}{9}+\frac{140 l_{2}}{9}+\frac{124 l_{2}^{2}}{9}\right)+\frac{1871 \pi^{6}}{540}\right]+C_{A}^{4}\left[\pi ^ { 2 } \left(\frac{257}{54}-\frac{512 \alpha_{4}}{9}+\frac{922 l_{2}}{27}-\frac{220 \zeta(3)}{9}\right.\right. \\
& \left.\left.-\frac{217 l_{2} \zeta(3)}{9}\right)+\pi^{4}\left(-\frac{25}{54}+\frac{20 l_{2}}{27}+\frac{31 l_{2}^{2}}{27}\right)+\frac{2897 \pi^{6}}{6480}\right] \text {, }
\end{aligned}
$$

with

$$
\frac{d_{A}^{a b c d} d_{A}^{a b c d}}{N_{A}}=\frac{N_{c}^{2}\left(N_{c}^{2}+36\right)}{24} .
$$

Numerical results of Eq. (A1) are given in Ref. [47]. All colour factors have been computed with the help of the program color [45].

[1] T. Appelquist and H. D. Politzer, Phys. Rev. Lett. 34 (1975) 43.

[2] W. Fischler, Nucl. Phys. B 129 (1977) 157.

[3] A. Billoire, Phys. Lett. B 92 (1980) 343.

[4] M. Peter, Phys. Rev. Lett. 78 (1997) 602 arXiv:hep-ph/9610209].

[5] M. Peter, Nucl. Phys. B 501 (1997) 471 arXiv:hep-ph/9702245.

[6] Y. Schroder, Phys. Lett. B 447 (1999) 321 arXiv:hep-ph/9812205.

[7] M. Melles, Phys. Rev. D 62 (2000) 074019 doi:10.1103/PhysRevD.62.074019 hep-ph/0001295.

[8] A. V. Smirnov, V. A. Smirnov and M. Steinhauser, Phys. Lett. B 668 (2008) 293 [arXiv:0809.1927 [hep-ph]].

[9] A. V. Smirnov, V. A. Smirnov and M. Steinhauser, Phys. Rev. Lett. 104 (2010) 112002 doi:10.1103/PhysRevLett.104.112002 [arXiv:0911.4742 [hep-ph]].

[10] C. Anzai, Y. Kiyo and Y. Sumino, Phys. Rev. Lett. 104 (2010) 112003 doi:10.1103/PhysRevLett.104.112003 arXiv:0911.4335 [hep-ph]].

[11] R. N. Lee and V. A. Smirnov, JHEP 1610 (2016) 089 doi:10.1007/JHEP10(2016)089 arXiv:1608.02605 [hep-ph]].

[12] C. Anzai, Y. Kiyo and Y. Sumino, Nucl. Phys. B 838 (2010) 28 Erratum: [Nucl. Phys. B 890 (2015) 569] doi:10.1016/j.nuclphysb.2010.05.012, 10.1016/j.nuclphysb.2014.11.025 arXiv:1004.1562 [hep-ph]].

[13] B. A. Kniehl, A. A. Penin, Y. Schroder, V. A. Smirnov and M. Steinhauser, Phys. Lett. B 607 (2005) 96 arXiv:hep-ph/0412083.

[14] T. Collet and M. Steinhauser, Phys. Lett. B 704 (2011) 163 doi:10.1016/j.physletb.2011.08.034 [arXiv:1107.0530[hep-ph]].

[15] C. Anzai, M. Prausa, A. V. Smirnov, V. A. Smirnov and M. Steinhauser, Phys. Rev. D 88 (2013) no.5, 054030 doi:10.1103/PhysRevD.88.054030 arXiv:1308.1202 [hep-ph]].

[16] T. Appelquist, M. Dine and I. J. Muzinich, Phys. Rev. D 17 (1978) 2074.

[17] A. Pineda and J. Soto, Nucl. Phys. Proc. Suppl. 64 (1998) 428 doi:10.1016/S0920-5632(97)01102-X hep-ph/9707481. 
[18] M. Beneke and V. A. Smirnov, Nucl. Phys. B 522 (1998) 321 doi:10.1016/S0550-3213(98)00138-2 hep-ph/9711391].

[19] M. Beneke, hep-ph/9806429

[20] B. A. Kniehl and A. A. Penin, Nucl. Phys. B 563 (1999) 200 arXiv:hep-ph/9907489.

[21] N. Brambilla, A. Pineda, J. Soto and A. Vairo, Nucl. Phys. B 566 (2000) 275 arXiv:hep-ph/9907240.

[22] N. Brambilla, A. Pineda, J. Soto and A. Vairo, Phys. Rev. D 60 (1999) 091502 arXiv:hep-ph/9903355.

[23] B. A. Kniehl, A. A. Penin, V. A. Smirnov and M. Steinhauser, Nucl. Phys. B 635 (2002) 357 arXiv:hep-ph/0203166].

[24] A. Pineda and J. Soto, Phys. Lett. B 495 (2000) 323 arXiv:hep-ph/0007197.

[25] N. Brambilla, A. Vairo, X. Garcia i Tormo and J. Soto, Phys. Rev. D 80 (2009) 034016 arXiv:0906.1390 [hep-ph]].

[26] A. Pineda and M. Stahlhofen, Phys. Rev. D 84 (2011) 034016 doi:10.1103/PhysRevD.84.034016 [arXiv:1105.4356][hep-ph]].

[27] M. Beneke and M. Steinhauser, Nucl. Part. Phys. Proc. 261-262 (2015) 378 doi:10.1016/j.nuclphysbps.2015.03.024 arXiv:1506.07962 [hep-ph]].

[28] M. Beneke, Y. Kiyo, P. Marquard, A. Penin, J. Piclum, D. Seidel and M. Steinhauser, Phys. Rev. Lett. 112 (2014) no.15, 151801 doi:10.1103/PhysRevLett.112.151801 [arXiv:1401.3005 [hep-ph]].

[29] M. Beneke, Y. Kiyo, P. Marquard, A. Penin, J. Piclum and M. Steinhauser, Phys. Rev. Lett. 115 (2015) no.19, 192001 doi:10.1103/PhysRevLett.115.192001 arXiv:1506.06864 [hep-ph]].

[30] A. A. Penin and N. Zerf, JHEP 1404 (2014) 120 doi:10.1007/JHEP04(2014)120 arXiv:1401.7035 [hep-ph]].

[31] C. Ayala, G. Cveti and A. Pineda, JHEP 1409 (2014) 045 doi:10.1007/JHEP09(2014)045 arXiv:1407.2128 [hep-ph]].

[32] M. Beneke, A. Maier, J. Piclum and T. Rauh, Nucl. Phys. B 891 (2015) 42 doi:10.1016/j.nuclphysb.2014.12.001 arXiv:1411.3132 [hep-ph]].

[33] Y. Kiyo, G. Mishima and Y. Sumino, Phys. Lett. B 752 (2016) 122 doi:10.1016/j.physletb.2015.11.040 arXiv:1510.07072 [hep-ph]].

[34] A. Bazavov, N. Brambilla, X. Garcia i Tormo, P. Petreczky, J. Soto and A. Vairo, Phys. Rev. D 90 (2014) no.7, 074038 doi:10.1103/PhysRevD.90.074038 arXiv:1407.8437 [hep-ph]].

[35] A. V. Smirnov, V. A. Smirnov and M. Steinhauser, Nucl. Phys. Proc. Suppl. 205-206 (2010) 320 doi:10.1016/j.nuclphysbps.2010.09.013 arXiv:1006.5513 [hep-ph]].

[36] A. V. Smirnov, V. A. Smirnov and M. Steinhauser, PoS RADCOR 2009 (2010) 075 arXiv:1001.2668 [hep-ph]].

[37] C. Anzai and Y. Sumino, J. Math. Phys. 54 (2013) 033514 doi:10.1063/1.4795288 arXiv:1211.5204 [hep-th]].

[38] Y. Sumino, arXiv:1607.03469 [hep-ph].

[39] R. N. Lee, Nucl. Phys. B 830 (2010) 474 doi:10.1016/j.nuclphysb.2009.12.025 arXiv:0911.0252 [hep-ph]].

[40] R. N. Lee and V. A. Smirnov, JHEP 1212 (2012) 104 doi:10.1007/JHEP12(2012)104 arXiv:1209.0339 [hep-ph]].

[41] R. N. Lee, J. Phys. Conf. Ser. 368 (2012) 012050 doi:10.1088/1742-6596/368/1/012050 arXiv:1203.4868 [hep-ph]].

[42] H. R. P. Ferguson and D. H. Bailey, "A Polynomial Time, Numerically Stable Integer Relation Algorithm", RNR Technical Report RNR-91-032, July 14, 1992.

[43] R. N. Lee and K. T. Mingulov, Comput. Phys. Commun. 203 (2016) 255 arXiv:1507.04256.

[44] D. Tulyakov2011, Proc. Steklov Inst. Math. 272 (2011) S162

[45] T. van Ritbergen, A. N. Schellekens and J. A. M. Vermaseren, Int. J. Mod. Phys. A 14 (1999) 41 doi:10.1142/S0217751X99000038 hep-ph/9802376.

[46] M. Prausa and M. Steinhauser, Phys. Rev. D 88 (2013) no.2, 025029 doi:10.1103/PhysRevD.88.025029 arXiv:1306.5566 [hep-th]].

[47] M. Prausa, Diplomarbeit, Karlsruhe Institute of Technology, 2013, (unpublished). 